\title{
3D PRINTING OF HOLLOW COMPOUNDS
}

\author{
Prepared by \\ Anil Kumar \& Ravi Katukam
}

\begin{abstract}
3D Printing is a process of manufacturing the product on the layer by layer. And it is best processes for producing a finished objects. It was started in 1980's with the invention of stereo lithography. It has gone through various ups and downs throughout its development and rejection of new technologies. In this paper, we h3ave summarized the printing of hollow compounds with different shapes. Applications of 3D printed hollow compounds are turbine blades, gears, disc brakes etc. One of the major application of 3D printing is hollow turbine blade. A turbine blade is the individual components. These are used to up the turbine section of a gas turbine. The blades must designed to withstand the high pressure and high temperature comings from combustor. These 3D printed hollow compounds has same strength as solid compounds when compared. Major advantages of the Hollow shaped compounds are heat transfer, low material cost. The aim of the paper is to reduce the cost of material and easy manufacturing of hollow compounds compared to traditional process and taken hollow turbine blade as example.
\end{abstract}

Keywords: hollow compounds, 3D printing, turbine blades, Additive manufacturing.

\section{INTRODUCTION}

Today's Engineers use highly sophisticated Computer Aided Design (CAD) and Computer Aided Engineering (CAE) software that allow them to design products that have far greater capabilities than products that were designed before their use. 3D printing allows complete geometry and property optimization no matter how organically or parametrically shaped the part needs to be. This is very understood in the design and optimization of hollow turbine blades used in aircrafts and any other hollow components. These hollow blades are designed to operate at high pressure and high temperature withstanding stress caused by centrifugal forces as well as large pressure forces. The turbine blades are specially designed to produce thrust from exhaust gases so they must be shaped in a specific aerodynamic form to translate incoming energy and pressure into thrust. To survive from these difficulties, materials like super alloys and many different methods of cooling's are used while blade manufacturing. The blade fatigue failure is one of the major failures in any steam turbines and gas turbines which is due to high dynamic stresses caused by blade vibration and resonance within the operating range of machine.

Failure Modes: Turbine blades are subjected to very tough environments inside the turbine. They face high temperatures, high stresses, and a potential environment of high vibration due to dynamic stresses. All three of these factors can lead to blade failure and potentially destroying the engine. Therefore turbine blades must be designed to resist these conditions. Turbine blades are subjected to stress from centrifugal force and fluid forces that can cause yielding, fracture or creep failures.

Problem Description: Many manufacturing companies using the Coordinate Measuring Machines (CMM) as their main Metrology tool for inspecting and qualifying any hollow products. However, while both software and hardware have advanced to aid the design and optimization of the process of designing and manufacturing, so we can do manufacturing of hallow turbine blade and other hallow compounds like gears, disc brakes in easy way. Many of the ideas for contact inspection have remained the same, i.e., the inspection process involves the measurement of individual points. This process is enough in many applications but for those involving parts that are designed specially to produce a certain fluid flow around its outer shell geometry and traditional manufacturing is time taking process for hollow compounds.

Motivation: Recent technologies in the fields of computeraided design and rapid prototyping (RP) have given designers the tools to rapidly generate an initial prototype. There are currently some different rapid prototyping technologies available, each with its own unique set of capabilities and restrictions. Rapid prototyping is very useful in the industry to minimize design cycles and improve the quality. The main advantage of layered 3D printing over conventional manufacturing is that complex hollow shapes can be physically realized without elaborate tooling. However, there are some specific part shapes like thin, slightly curved shell-type, hallow structures (turbine blades, disc brakes, gears etc.) where the application of 3D printing is poorly suited and may result in lack of strength, stair-step effect or large number of layers, resulting in higher build time but with the todays available technology in the $3 \mathrm{D}$ printing we can avoid these problems And make metal 3D printed compounds also.

Literature Survey: R. Anita, S. Arunachalamm, P.Radhakrishnan [1], Rapid prototyping (RP) meets the current needs in the industry to minimize design cycles and improve the quality. Fused deposition modeling (FDM) is one of the major technologies of RP. Various process parameters used in FDM effect the quality of the prototype. Work was undertaken to evaluate the influence of the 
parameters on the quality features of the prototypes.This paper contains the results of the study and the conclusions arrived from it.

Jack G.Zhou, Daniel Herscovici and Calvin C.Chen [2], 'Parametric Process Optimization to improve the Accuracy of Rapid Prototyped Parts', USA. The main use of a rapid prototyping system are speed and accuracy and user selected manufacturing. A specially designed model with twenty dimensional, geometrical, and surface roughness features has been used in the inspection of Rapid prototyping manufacturing process. Two analysis tools, response surface methodology and Analysis of Variance have been used to evaluate the Stereo lithography Rapid prototyping process and to perform the product optimization. The optimal setups of Stereo lithography manufacturing parameters for both individual features and a general part with various features have been concluded from this study.

Deepika Jijotiya and Dr. PrabhuLalVerma [3] Department of Mechanical Engineering, Samrat Ashok Technological Institute, Vidisha, M.P., India. Rapid prototyping is a one of the techniques used to quickly fabricate physical parts or assembly using 3D computer aided design data. Construction of the parts or assembly is usually done using 3D printing. This technique used for a much wider range of applications. They are even used to manufacture productionquality parts in less numbers. Rapid application developmentis a software development methodology that used minimize the planning in rapid prototyping. The "planning" of software developed using Rapid application development is interleaved with writing the software itself. The lack of preplanning allows software to be written much faster and makes it easier to change requirements. In this paper we discussed a survey of performance based advanced rapid prototyping techniques. The performance based techniques are categorized based upon different ways. We also analysis the major improvement in recent methods advanced rapid prototyping techniques.

\section{Analysis of Turbine Blades}

Fem of Turbine Blades: Turbines blades are subjected to very particular loads and stresses due to the nature of wind and loads are highly variable. These Varying loads are more difficult to handle than static loads. This because the material becomes fatigued. Moreover due to low density of air medium surface required more capturing energy. When designing a hollow turbine blades, the aim is to attain the highest possible power output as solid turbine blade under particular atmospheric conditions and this depends on the shape of the blade.

In this paper it is given that the turbine blades are act as cantilever beams because one end of the turbine blades connected to shaft. Hollow compounds with different shapes made of shell element is used in this the geometry of blade is modelled in ANSYS to obtain the required properties of the blade and position of spars with all material properties. We have taken that all compounds with different shapes are cantilever beams. The following are the comparison of solid and hollow components of different shapes of structural analysis are listed below.

\section{Analysis report:}

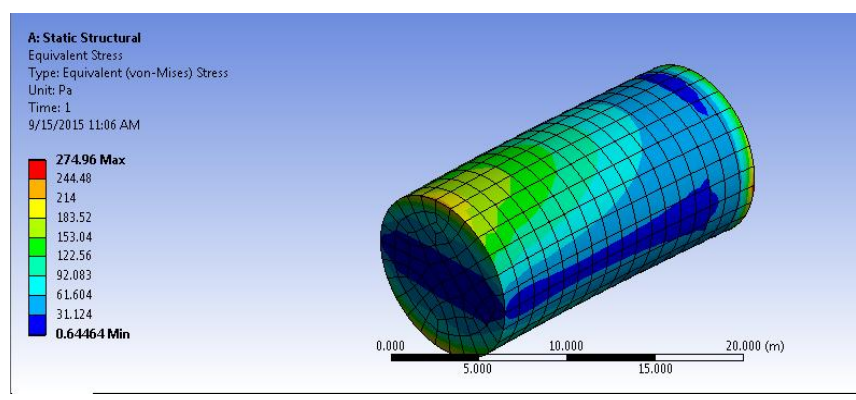

Figure 1) Equivalent stresses of solid section of circular model

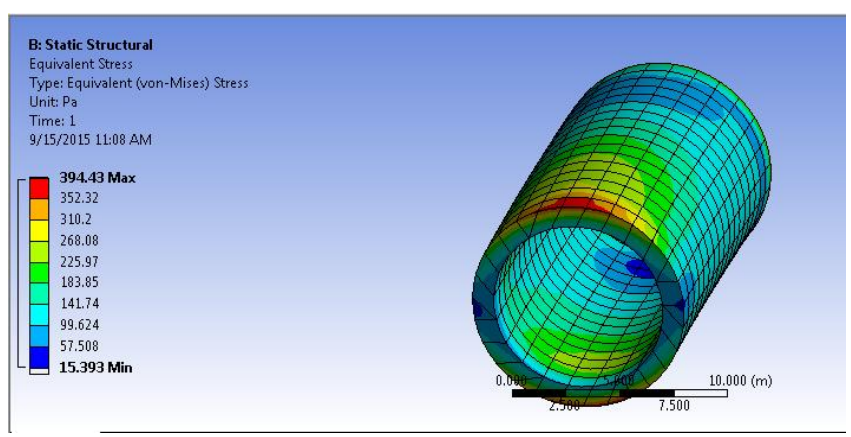

Figure 2) Equivalent stresses of hollow section of circular shape.

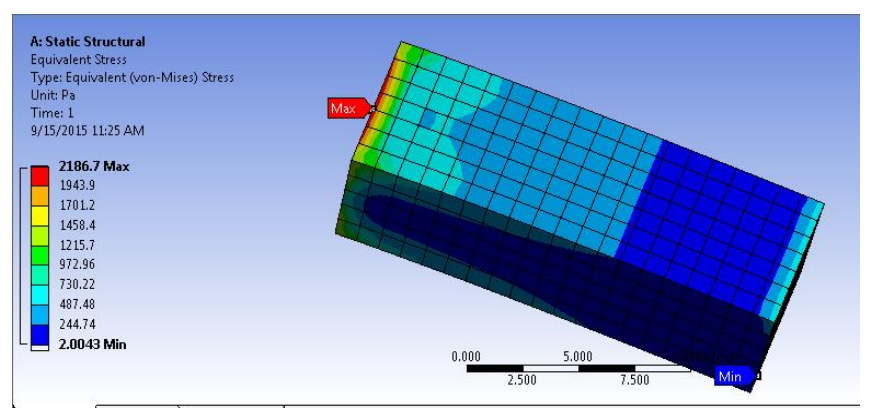

Figure 3) Equivalent stress of solid section of rectangular shape

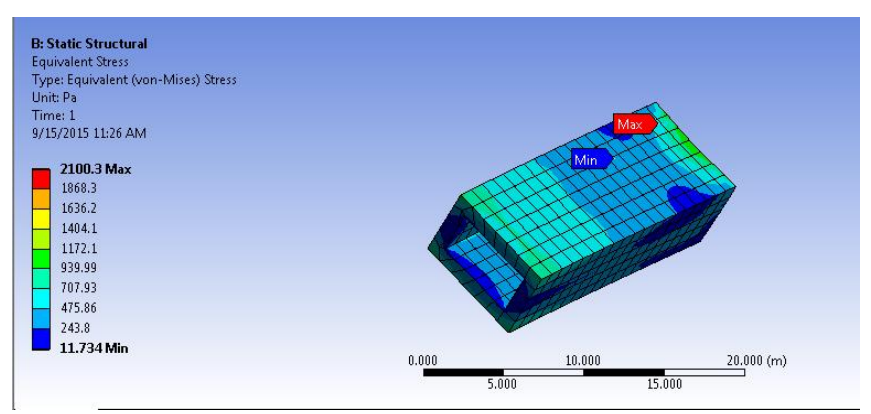

Figure 4) Equivalent stresses of hollow section of rectangular shape. 


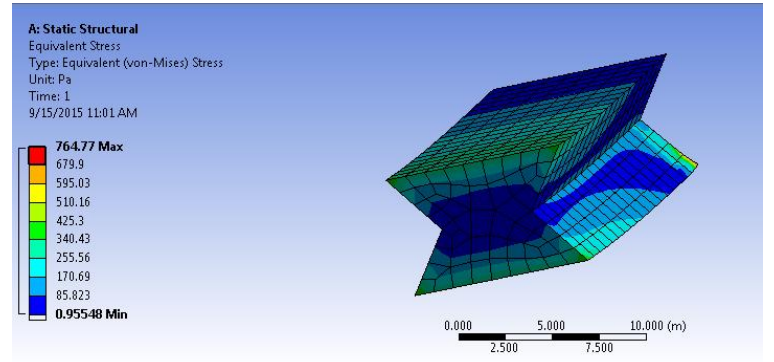

Figure 5) Equivalent stresses of solid section of $x$ shape

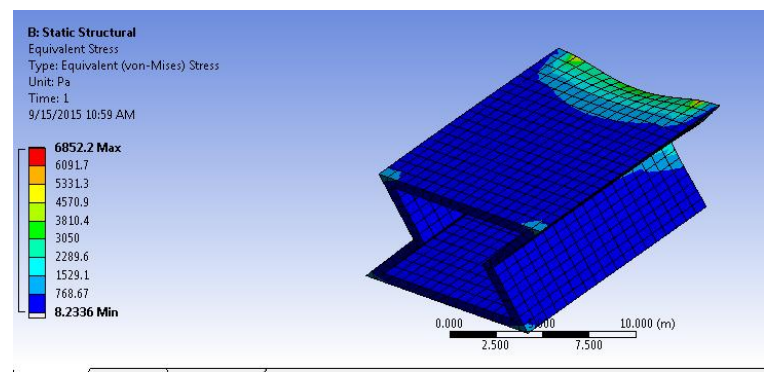

Figure 6) Equivalent stresses of hollow section of x shape

Table: Comparison of stress

\begin{tabular}{|l|l|l|}
\hline Shape & $\begin{array}{l}\text { Solid (max } \\
\text { equivalent } \\
\text { stress) }\end{array}$ & $\begin{array}{l}\text { Hollow (max } \\
\text { equivalent stress) }\end{array}$ \\
\hline Circle & $274.96 \mathrm{~Pa}$ & $394.43 \mathrm{~Pa}$ \\
\hline Rectangular & $2186.7 \mathrm{~Pa}$ & $2100.3 \mathrm{~Pa}$ \\
\hline X shape & $764.77 \mathrm{~Pa}$ & 685.2 \\
\hline
\end{tabular}

Scope of this work: The concept of $3 \mathrm{D}$ printing is purely different from traditional manufacturing. This paper gives use of $3 \mathrm{D}$ printing in manufacturing of hollow compounds with different shapes in many applications. Major application is turbine blades and there are other applications such as gears, disc brakes etc.

$\checkmark$ The scope this work is if the desired component contains any hollow shapes it is difficult to manufacture in traditional way where as in 3-D printing does very easy.

$\checkmark$ Coolants can be placed in space between hollow compounds to reduce heat transfer.

$\checkmark \quad$ Wastage of material can be reduced.

$\checkmark$ Cost of 3D Printing is less as compared to traditional manufacturing.

Our main scope is to find the dimensional accuracy of the $3 \mathrm{D}$ printed components.

Result: The finite element analysis for structural analysis is carried out. The structural analysis was performed for different shapes of solid and hollow components with the volume remaining same and the results obtained in the both the cases when compared are approximately same in hollow shapes components compared to solid shapes component.

\section{CONCLUSION}

The technologies provide highest quality in design prospects which ensures better results, convenience and moreover a perfect design. The very nature of $3 \mathrm{D}$ printing is creating a part layer by layer, instead of traditional methods of manufacturing lower costs in raw material. The stress analysis when performed on the both solid and hollow shapes components the results is approximately same and there is a scope of fixing coolant in between the hollow space so that heat transfer can be reduced.

\section{REFERENCES:}

[1] Habali, S.M.; Saleh, I.A. Local design, testing and manufacturing of small mixed airfoil wind turbine blades of glass fiber reinforced plastics Part I: Design of the blade and root. Energy Convers. Manag.2000, 41, 249-280.

[2] Thresher, R.W.; Dodge, D.M. Trends in the evolution of wind turbine generator configurations and systems. Wind Energy 1998, 1, 70-86.

[3] Ahlstrom, A. Emergency stop simulation using a finite element model developed for large blade deflections. Wind Energy 2006, 9, 193-210.

[4] S.Gowreesh, N.Sreenivasalu Reddy and N.V.Yogananda Murthy. "Convective heat transfer analysis of aero gas turbine blade using ansys", International journal of Mechanics of solids, vol4, No.1, March 2009(ppt55-62).

[5] P.Kauthalkar, Mr.Devendra S.Shikarwar, and Dr.Pushapendra Kumar Sharma. "Anlysis of thermal stresses distribution pattern on gas turbine blade using ansys", International journal of Engineering Education and technology, Vol.2, No.3, Nov 2010.

[6] John.V, T.Ramakrishna. "The design and analysis of gas turbine blade", International Journal of Advanced Research and Studies, Vol 2, No.1, Dec 2012.

[7] V.Raga Deepu, R.P.Kumar Ropichrla. "Design and coupled field analysis of first stage gas turbine rotor blades", International journal of Mathematics and Engineering, Vol 13, No.2, Pages: 1603-1612.

[8] V. Veeraragavan Effect of Temperature Distribution in 10c4/60c50 Gas Turbine Blade Model Using Finite Element Analysis.

[9] V.R.S.M. Kishore Ajjarapu1, K. V.P.P.Chandu2 D.M.Mohanthy Babu3 "design and analysis of the impeller of a turbocharger for a diesel engine".

[10]R D V Prasad1, G Narasa Raju2, M S Srinivasa Rao3, N Vasudeva Rao4 Steady State Thermal \& Structural Analysis of Gas Turbine Blade Cooling System.

[11] Timoshenko, "STRENGTH OF MATERIALS PART 1 " third edition.

[12] Jack D Mattingly, "Elements of Propulsion: Gas Turbine and Rockets".

[13] H.S.Manohar, Finite Element Method, first edition.

[14] Singiresu S. Rao, "The Finite Element Method in Engineering".

[15] Raghevandra Panchal, 'Unmanned Aerial Vehicle RC Quad copter', CHARUSAT, Gujarat. 
[16] R. Anita, S. Arunachalamm, P.Radhakrishnan, 'Critical Parameters Influencing the Quality of Prototype in Fused Deposition Modeling', Journal of Processing Technology.

[17] Dr. Mark Reeder, International Journal of Micro Air Vehicles

[18] Rajan Bansal, 'Improving dimensional accuracy of fused deposition modeling (FDM) parts using response surface methodology', National Institute of Technology, Rourkela.

[19] AshutoshChouksey, 'Study of parametric optimization of fused deposition modeling process using response surface methodology' National Institute of Technology, Rourkela.

[20] Jack G.Zhou, Daniel Herscovici and Calvin C.Chen, 'Parametric Process Optimization to improve the Accuracy of Rapid Prototyped Parts', USA.

[21] NASA Engineers Build Aircraft Using 3D Printed Parts and Harvested UAV Components.

[22] Introduction to Rapid Prototyping Technology and Advancements of Material \& Machine for Fused Deposition Modelling Technique. 